\title{
Thermal Conductivity and Contact Conductance of BSCCO-2212 Material
}

\author{
Suntao Yang, Binjiang Chen, Eric E. Hellstrom, Eric Stiers and John M. Pfotenhauer \\ Applied Superconductivity Center, University of Wisconsin - Madison, Madison, WI 53706
}

\begin{abstract}
Thermal conductivity of bulk high temperature superconducting materials is a very important property in the current lead applications because it determines the heat load of the lead at the low temperature end. The thermal conductivity of BSCC0.2212 superconducting material has been measured between 10 and $130 \mathrm{~K}$. Our results compare favorably with other published results. The thermal contact conductance between a joint of BSCCO-2212 and copper has also been measured. Our results indicate that although this kind of joint provides a relatively poor thermal interface, the electrical resistance is small. This joint is still a good choice for making a easy joint which requires both thermal and electrical interfacing.
\end{abstract}

\section{I . Introduction}

Soon after the discovery of high temperature superconducting materials, it was realized that they have advantages as current leads for conventional superconducting magnets operating at liquid helium temperature. The objective of a current lead design is to minimize the heat leak introduced by the transmission of a given current into the cryostat. This heat comes from two sources: it is conducted down the lead from room temperature and it is generated within the lead by ohmic loss. One therefore wishes to minimize both the thermal conductivity and electrical resistance of the lead material. For a high temperature superconducting current lead, the material of the upper stage (between the intermediate temperature and the room temperature) is usually conventional copper while superconducting material is used in the lower stage. When this lead is operating in the superconducting state, no heat is generated in the lower stage. Because of the relatively low thermal conductivity as compared to metals and no heat is generated in the superconductor part of the lead, high temperature superconducting current leads have the potential to reduce the heat load at liquid helium temperature to $1 / 5$ of that of the best possible conventional current leads. Numerous investigations have been carried out in the last few years to address this possibility [1], [2].

The heat conduction through a high temperature superconductor which spans the temperature range from $T_{0}$ to

Manuscript received October 18, 1994.

This work sponsored by the Defense Nuclear Agency under DNA MIPR 92-719, and Work Unit CD:00014, RCC: 7010
$4.2 \mathrm{~K}$ is given by

$$
\mathrm{Q}=\frac{\mathrm{A}}{\mathrm{L}} \int_{4.2 \mathrm{~K}}^{\mathrm{T} 0} \mathrm{kdT}
$$

where $A$ is the cross section area and $L$ is the length of the conductor, $\mathbf{k}$ is the thermal conductivity of the material, and $\mathrm{T}_{0}$

where $\Gamma\left(T_{0}, 4.2 \mathrm{~K}\right)=\int_{4.2 \mathrm{~K}} \mathrm{kdT}$ is called the thermal conductivity integral. For a given operating current, the heat load may be reduced by either a decrease in the thermal conductivity integral $\Gamma$, or by an increase in the critical current density, since in the later case a fixed current will allow a decrease in area $A$.

Thermal conductivity of high temperature superconducting materials have been reported in many papers [1], [2], [4], [6]. The typical values for thermal conductivity integral are as follows: for sintered BSCCO-2223 material, $\Gamma(77 \mathrm{~K}, 4.2 \mathrm{~K})=$ $80 \sim 130 \mathrm{~W} / \mathrm{m}$; for melt cast BSCCO-2212 material, $\Gamma(77 \mathrm{~K}$, $4.2 \mathrm{~K})=103 \mathrm{~W} / \mathrm{m}$; for sintered YBCO material, $\Gamma(77 \mathrm{~K}, 4.2 \mathrm{~K})$ $=234 \mathrm{~W} / \mathrm{m}$. Samples prepared by different processing methods usually show similar thermal conductivity results, although other properties may be very different. In comparison, for electrolytic tough pitch annealed copper (RRR $=10$ ), a typical conventional current lead material, the thermal conductivity integral, $\Gamma(77 \mathrm{~K}, 4.2 \mathrm{~K})=3.7 \times 10^{5}$ $\mathrm{W} / \mathrm{m}$, which is several orders of magnitude larger than that of the high temperature superconducting materials.

Besides thermal conductivity and critical current density, the joint connection between the high temperature superconductor and copper is also critical in current lead designs. For the currents typically required, large electrical contact resistance can limit the current being transported, and provide large heat load to the system. On the other hand, as most high temperature superconducting material in the current lead is cooled at least partially by conduction, thermal contact resistance between the high temperature superconductor and the copper plate providing cooling will determine the temperature profile of the superconductor. A large thermal contact resistance may result in poor cooling for the superconductor, and therefore degrade current carrying capacity. In addition to the electrical properties, it is often very useful to know the thermal properties of the joint.

In this paper, thermal conductivity and critical current density of BSCCO-2212 prepared for current leads applications as well as the thermal and electrical contact 
conductance of a joint between BSCCO-2212 and copper plate are reported.

II. Sample preparation and experimental set-up

The high temperature superconducting rods were made from nominal composition of $\mathrm{Bi}_{2} \mathrm{Sr}_{2} \mathrm{Ca}_{1} \mathrm{Cu}_{2} \mathrm{O}_{\mathrm{y}}$ (BSCCO-2212) by melt processing. The starting BSCCO-2212 powder was purchased from SSC, Inc. at a purity of $99.9 \%$. The powder was mixed with $4 \mathrm{wt} . \%$ silver, packed into a silver tube or silver boat, then heated to and held at $908-910^{\circ} \mathrm{C}$ for about 20 minutes to obtain partial melting of the powder. The sample was then cooled to a lower temperature ranging from 820 $860^{\circ} \mathrm{C}$ and held for extended period of time to allow the reformation of the BSCCO-2212 phase. This period was typically between 12 to 50 hours. The sample was subsequently cooled to room temperature. Round or square shaped BSCCO-2212 rods were obtained by peeling off silver from the rod. These samples had a density in the range of 5.877 - $6.204 \mathrm{~g} / \mathrm{cm}^{3}$.

The two samples used for thermal conductivity measurements were cylindrical, with a diameter of $5.89 \mathrm{~mm}$ and length of $29.67 \mathrm{~mm}$ and $23.72 \mathrm{~mm}$ respectively. The thermal conductivity of the BSCCO-2212 bulk samples was measured over the temperature range from $10-130 \mathrm{~K}$ using a steady state heat flow method. One end of the sample was attached to the copper sample holder of a Supertran Refrigerator (Janis Research Company, Inc.). The temperature of the sample holder was controlled by the standard feedback method utilizing a calibrated thermometer. It uses controlled cooling by liquid helium and heating through an electrical resistance heater. This temperature can be easily adjusted over the range from $4.2 \mathrm{~K}$ to $300 \mathrm{~K}$. A second heater was attached to the other end of the sample through a small copper block. The heat flow was applied along the longitudinal direction of the sample through this heater, and the temperature gradient of the sample was measured with silicon diode temperature sensors (Lake Shore Cryotronics, Inc.), which were individually calibrated to an accuracy of $\pm 50 \mathrm{mK}$. Fig. 1 shows the set up for thermal conductivity and thermal contact conductance measurement.

The critical current density of the samples was measured using the standard four-lead method using the criterion of 1 $\mu \mathrm{V} / \mathrm{cm}$. The connection between the BSCCO-2212 and copper was achieved by an interface of silver and "woods" metal (Williams-Collins), as shown in fig. 2. This commercially available "woods" metal is an alloy of bismuth, tin, lead and cadmium with melting point of $70^{\circ} \mathrm{C}$. A small part $(\sim 4 \mathrm{~mm}$ length) of the original silver tube was left on the high temperature superconductor to provide a connection interface, when the rest of the tube was peeled off. Braided copper wires were then soldered on the silver tube using low temperature solder "woods" metal. This connection provides a strong physical connection between the high temperature superconductor and the copper. For the critical current measurement, additional voltage taps were installed at both ends of each joint to measure the contact resistance. The contact surface area was $1.32 \mathrm{~cm}^{2}$. This kind of joint is very easy to make, solidifies quickly. The mechanical strength of the joint was strong enough to support the sample and a small copper block in the test facility.

III Results and Discussions

\section{A. Thermal conductivity}

In the steady state heat flow method, the relationship between the heat flux $Q$ and thermal conductivity $k$ of a uniform material is given by $Q=k A \Delta T / \Delta L$, where $A$ is the cross section area of the sample and $\Delta \mathrm{T}$ is the temperature difference along the sample in a distance $\Delta L$. In our experiment set up, the error in determining $\mathbf{k}$ was mainly due to the error in determining $\Delta T$, because $Q, A$, and $\Delta \mathrm{L}$ can be measured very precisely. In our tests, the error in temperature determination caused by thermal contact resistance was eliminated by the placement of the thermometers, so the error in temperature measurement was due solely to the accuracy of the thermometers. As each thermometer was individually

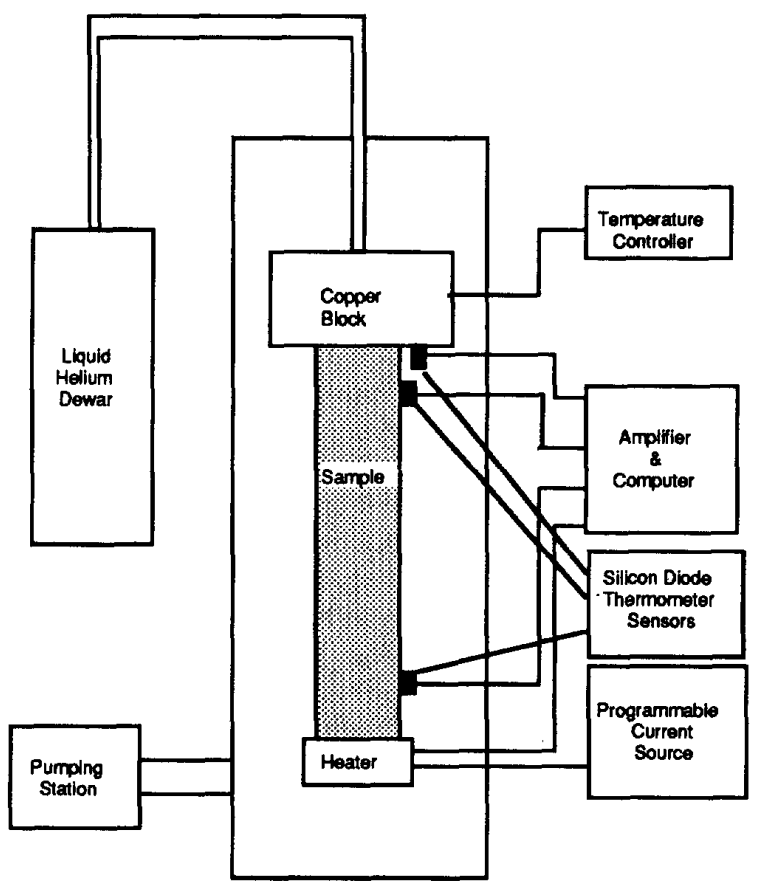

Fig. 1. Schematic diagram of thermal conductivity and thermal contact conductance measurement device using steady state heat flow method. 


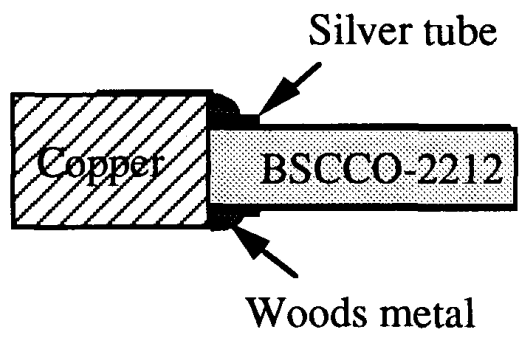

Fig. 2. Schematic diagram of BSCCO-2212 and copper joint.

calibrated to an accuracy of $50 \mathrm{mK}$, and $\Delta \mathrm{T}$ was in the range of $2 \sim 8 \mathrm{~K}$, our calculation shows this would result in an uncertainty of $6 \%$ for the thermal conductivity results.

Fig. 3 shows the thermal conductivity data for one of the BSCCO-2212 samples, which has a transition temperature of $92 \mathrm{~K}$. Although the highest critical current density measured in these samples to date is $430 \mathrm{~A} / \mathrm{cm}^{2}$ at $77 \mathrm{~K}$, the $J_{\mathcal{C}}$ for the sample used in these thermal conductivity measurements were only 144 and $43 \mathrm{~A} / \mathrm{cm}^{2}$ respectively, because sample with the highest $\mathrm{J}_{\mathrm{C}}$ value was broken. Thermal conductivity results of these two samples are very close. As the temperature is decreased from $130 \mathrm{~K}$, thermal conductivity decreases, and reaches a minimum around $90 \mathrm{~K}$. It then increases before reaching a peak around $45 \mathrm{~K}$ and falls off at low temperatures. It is interesting to note that although the tested samples only have $\mathrm{J}_{\mathrm{C}}$ of 144 and $43 \mathrm{~A} / \mathrm{cm}^{2}$, the measured thermal conductivity data compare favorably with published data for BSCCO-2212 materials with similar density, but much higher $\mathrm{J}_{\mathrm{C}}$ values [3].

The thermal conductivity measured experimentally is the sum of conduction due to the charge carriers and due to the

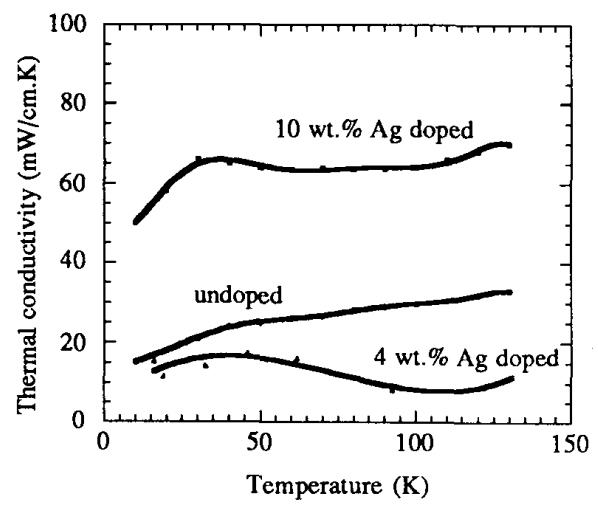

Fig. 3. Temperature dependence of thermal conductivity of BSCCO-2212 superconductor. Data for the undoped and $10 \mathrm{wt} . \%$ Ag doped samples are from references [1], [4]. phonons. The charge carrier component of thermal conductivity normally decays as superconducting pairs are formed because these pairs do not transport energy. On the other hand, since paired carriers no longer scatter with phonons, the lattice thermal conductivity usually increases below $T_{c}$. The anomaly that thermal conductivity reaches a peak below $T_{c}$ before falling off at low temperature is similar to that reported by other investigations [1], [4]. However, it is believed that this was not associated with the $T_{c}$ or the superconducting state. Rather, it is believed to be related to a Ag doping effect, because the enhancement of the thermal conductivity was qualitatively similar to that of pure silver. It was reported that for samples which do not contain silver, the thermal conductivity decreases monotonically as the temperature is lowered [4]. The anomaly was only observed for samples with 10-15 wt.\% silver doping. Our results show that the anomaly is present even for BSCCO-2212 samples doped with $4 \mathrm{wt}$. \% silver.

For BSCCO-2212 used in current lead applications, it is desired that the material have the lowest possible thermal conductivity, with the largest possible $\mathrm{J}_{c}$. The thermal conductivity results reported here are comparable to thermal conductivity of samples not containing silver, which are much lower than that of samples doped with 10-15 wt.\% silver. These indicate that doping BSCCO-2212 with up to 4 wt.\% silver does not degrade the thermal performance of this material.

\section{B. Thermal contact conductance}

Many studies have measured the electrical contact resistance between joints of high temperature superconductor and copper. However, little has been reported for thermal contact resistance between high temperature superconductor and copper. The thermal conductance per unit area of bonding $\left(\mathrm{K}_{\mathrm{b}}\right)$ between copper and BSCCO-2212 can be determined by the classical steady state method: a small heat flux $Q\left(\mathrm{~W} / \mathrm{m}^{2}\right)$ transmits through the joint, a temperature difference $T_{1}-T_{0}$ is established between the upper end of the high temperature superconductor sample (at $\mathrm{T}_{1}$ ) and the copper block (at $\mathrm{T}_{0}$ ). $\mathrm{K}_{\mathrm{b}}$ is then defined by the relation $\mathrm{Q}=\mathrm{K}_{\mathrm{b}}(\mathrm{T})\left(\mathrm{T}_{1}-\mathrm{T}_{0}\right)$, where $\mathrm{T}=\left(\mathrm{T}_{1}+\mathrm{T}_{0}\right) / 2$ is the mean temperature of the joint. Fig. 4 shows the thermal contact conductance for the BSCCO-2212 and copper joint described in fig. 2 .

Temperature dependence of the thermal conductance of the joint has a similar general shape as the thermal conductivity results. Starting at $130 \mathrm{~K}$, thermal conductance decreases before increasing to a maximum around $45 \mathrm{~K}$, then dropped off at low temperatures. This feature may be due to the fact that we used silver and "woods" metal as the interface between BSCCO-2212 and copper. As in the case for thermal conductivity, the anomaly in the temperature dependence may be due to the silver.

The measured thermal contact conductance is much lower 


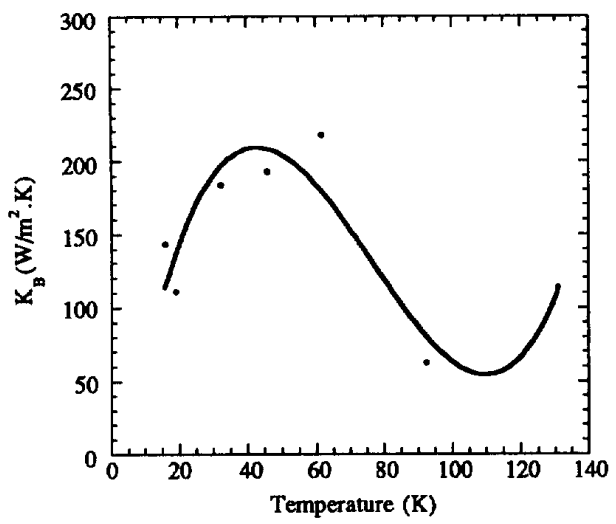

Fig. 4. Thermal contact conductance of the joint between BSCCO-2212 and copper as a function of temperature.

than that reported for copper-cyanoacrylate adhesive YBCO joints. This is may be due to the fact that "woods" metal is a relatively poor thermal interface compared to the adhesive. However, if the joints are also used for transferring electrical current, then the "woods" metal solder joints have the advantages. Our measurements show that the electrical resistance of such a joint is $11.5 \mu \Omega$.cm ${ }^{2}$ at $77 \mathrm{~K}$. This was comparable to many of the reported contact resistance results $[6],[7]$.

\section{Conclusions}

We have measured the thermal conductivity of superconducting BSCCO-2212 materials with 4 wt.\% silver doping. The results show a similar temperature dependence with samples that have much higher silver doping. However, the magnitude of thermal conductivity for these samples is comparable to that of undoped materials, which is much lower than that of samples with $10 \mathrm{wt} . \%$ or more silver.
The joint between BSCCO-2212 and copper using silver and "woods" metal as interface shows low electrical resistance. The temperature dependence of thermal conductance of these joints has a shape similar to the temperature dependence of the thermal conductivity of BSCCO-2212. This dependence may be due to the presence of silver in both cases. Although this kind of joint provides a relatively poor thermal interface, it is still a good choice when an easy to make joint is required for both thermal and electrical interfacing.

\section{References}

[1] P. F. Hermann et al., "European project for the development of high $T_{c}$ current leads", IEEE Trans. on Applied

Superconductivity Vol. 3, 1993, page 876-880.

[2] J. R. Hull, "High-Temperature Superconducting Current Leads", IEEE Trans on Applied Superconductivity, Vol. 3, 1993, page 869-875.

[3] J. Bock, private communication.

[4] M. Matsukawa, F. Tatezaki, K. Noto, H. Fujishiro, K. Michishita and Y. Kubo, "Thermal conductivity of Agdoped Bi-2212 superconducting materials prepared by the floating zone method", Crogenics, Vol. 34, 1994, page 685-688.

[5] N. Wendling, J. Chaussy, J. Mazuer and J. Odin, "Thermal conductance of copper-cyanoacrylate adhesive YBCO joints between 5 and $300 \mathrm{~K}$ ", Cryogenics, Vol. 34, 1994, page 89-90.

[6] F. Grivon, A. Leriche, C. Cottevieille, J.C. Kermarrec, A. Petitbon, A. Fevrier, "YBCO current lead for liquid helium temperature applications", IEEE Trans. on Magnetics, Vol. 27, 1991, page 1866-1869.

[7] R. C. Niemann, Y. S. Cha, J. R. Hull, W. E. Buckles and M. A. Doughty, "Performance evaluations of demountable electrical connections", Advances in Cryogenic Engineering, New York: Plenum Press, 1994, Vol.39. pp1153-1160. 